\title{
Plate-forme PROMOCO (plate-forme Robotique Mobile Communicante) Présentation des impacts de formations, de prestations industrielles et de recherche
}

\author{
Valéry BOURNY 1,2, Thierry CAPITAINE1,2, Jacky SENLIS2, Mohamed. HAMZAOUI2, \\ Aurélien LORTHOIS2, Ludovic DUVAL2, Université de Picardie Jules Verne - Amiens \\ 1Laboratoire Modélisation, Information et Systèmes (MIS) - Amiens \\ 2INstitut Supérieur des Sciences Et des Techniques (INSSET) - Saint-Quentin
}

\section{MOTS CLEFS}

Plate-forme technologique, Robotique mobile, Architecture réseau, Capteurs et actionneurs intelligents

\section{RESUME}

L'Insset de Saint Quentin est un institut de l'Université de Picardie Jules Verne qui propose une formation Master spécialisée dans les systèmes embarqués dans les transports. Cette formation s'appuie sur la présence d'une petite équipe d'enseignants chercheurs du laboratoire MIS (Modélisation, Informatique et Systèmes) de l'UPJV privilégiant les activités d'innovations et transferts technologiques dans des domaines pluridisciplinaires (micro électronique, physique, énergie). Les problématiques traitées concernent généralement la localisation et la conception de capteurs/actionneurs intelligents pour des systèmes mobiles autonomes. Des solutions sont développées par les étudiants et les enseignants chercheurs pour répondre aux besoins de laboratoires de recherche, d'industriels et de formations. Cet article décrit l'historique, la structure de la plateforme et les avantages de son utilisation pour la convergence des activités de recherche, de formation et d'innovation industrielle. Cette plateforme a été labellisée par le pôle de compétitivité international ITRANS en 2007.

\section{Introduction}

\subsection{Le contexte}

La formation Systèmes Embarqués dans les Transports (SET) de l'Insset [1] comprend 3 années universitaires (licence 3 et Master Professionnels). Elle repose sur une maîtrise de 4 disciplines principales : les FPGA, le traitement du signal, la programmation de systèmes d'exploitation temps réel et la conception de cartes à base de microcontrôleurs et DSP. D'autres modules sont proposés en option en lien direct avec les différentes spécialités des systèmes embarqués. Les enseignants mettent particulièrement l'accent sur la mise en application concrète de ces enseignements théoriques par le biais de projets gérés par des groupes d'étudiants sous la responsabilité d'un enseignant chercheur. Ces projets sont très importants pour la validation du diplôme car ils représentent environ $30 \%$ de la note finale en formation continue (jusqu'à 10 crédits ECTS pour les M2). Les sujets sont proposés au mois de septembre et concernent le développement de solutions aux problématiques des entreprises, la réalisation de démonstrateurs à des fins de veilles technologiques ou pour répondre aux besoins des laboratoires. Les enseignants chercheurs de l'Insset intervenant dans cette formation sont membres du thème perception en robotique du laboratoire MIS [2] (perception de l'environnement, localisation, la vision omnidirectionnelle, les robots mobiles et aériens). Nos travaux de recherche [3], [4] et de prestations industrielles nous amènent généralement aux développements de prototypes, la mise en place d'essais expérimentaux et de validation, auxquels participent les étudiants de la formation SET. 


\subsection{Constat}

Le bilan de cette démarche, réalisé il y a quelques années, met en évidence un intérêt pour l'insertion professionnelle de nos étudiants mais elle n'est pas efficace pour la valorisation universitaire ni pour la pérennité des solutions et prototypes développés :

$>$ Pas d'ouverture aux traitements de problématiques de recherches transverses.

De nombreux démonstrateurs industriels dédiés à une application spécifique.

Une approche événementielle non pérenne des développements effectués.

Exploitation des investissements (financiers et humains) non optimisée.

Mise en démonstration rapide d'un prototype difficile.

Aucune mutualisation et interaction des ressources pour la formation, la recherche et l'industrie.

\subsection{La solution : Une plate forme technologique?}

Les problématiques généralement traitées par le laboratoire concernent la conception de solutions pour répondre aux différentes problématiques des robots autonomes et AGV (Automatic Guided Vehicles). On y retrouve de nombreuses spécialités telles que les capteurs /actionneurs intelligents, leurs réseaux associés, la perception de l'environnement, la gestion de flottes de mobiles, etc. Nous nous sous sommes donc logiquement orientés vers une plateforme composée de 2 robots mobiles communicants: PRoMoCo pour " Plateforme Robotique Mobile Communicante ». Cette plateforme devait être :

$>$ composée de briques logicielles et matérielles autonomes communicant sur le bus CAN.

$>$ évolutive et durable.

$>$ opérationnelle en tant que démonstrateur de solutions innovantes pour les industriels et pour nos activités de recherche.

$>$ directement exploitable par un industriel ou un enseignant chercheur pour valider rapidement les fonctionnalités d'une brique, d'un algorithme ou d'une supervision.

\subsection{Les ressources}

\section{Ressources financières}

Cette plateforme a été financée pour un montant de 50K€ par :

Le dispositif Interreg [5] dans le cadre de formations pour des étudiants francophones

Les fonds FSI de la région Picardie

Les fonds propres de l'Insset

\section{$\underline{\text { Ressources matérielles }}$}

Les développements sont assurés par les étudiants de la formation SET qui disposent d'un logiciel de conception et simulation de schémas électroniques, d'une station de maintenance CMS, d'un banc de réalisation des circuits imprimés et par les étudiants de l'IUT de l'Aisne pour la conception et la réalisation des parties mécaniques. L'Insset a mis à notre disposition une salle dédiée (figure 1.) 


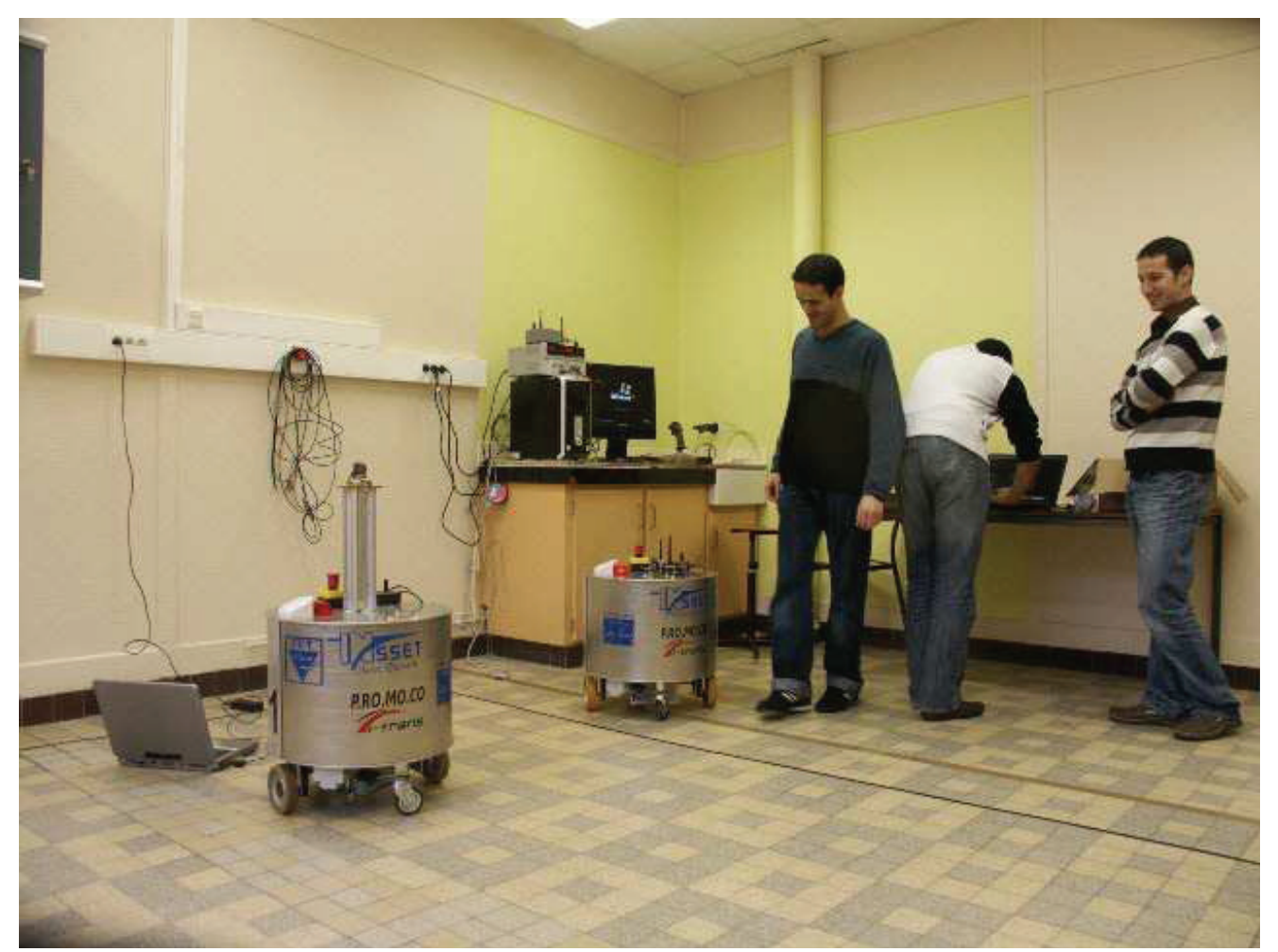

Figure 1. Salle dédiée avec des étudiants SET

\section{Les projets étudiants}

Le développement des briques repose plus particulièrement sur les projets étudiants. Des groupes d'étudiants sont constitués lors de la rentrée de septembre. Chaque groupe a en charge le développement d'une brique spécifique, la rédaction du cahier des charges, les phases de tests et de validation ainsi que de la rédaction d'un document technique et d'utilisation. Généralement un étudiant de M2 encadre des étudiants de M1 et de L3. C'est cet étudiant de M2 qui sera le seul interlocuteur vis-à-vis de l'équipe enseignante et qui doit assurer l'organisation du projet avec les membres de son équipe en intégrant toutes les contraintes de réalisation. Les étudiants disposent d'environ 12 heures hebdomadaires pour travailler durant le premier semestre, 2 réunions par mois sont planifiées avec l'équipe enseignante. Environ 8 projets par année sont consacrés au développement de cette plateforme.

\section{La plateforme technologique}

\subsection{Structure de la plateforme}

Cette plateforme technologique est constituée d'une flotte de robots mobiles (actuellement 2) qui peut être gérée par un superviseur déporté (communication via un réseau sans fils) ou intégrée à un robot particulier. Chaque robot est équipé de deux roues motrices et d'une roue folle à l'avant. Des codeurs optiques assurent les fonctions d'odométrie pour l'asservissement en vitesse et en position. Nous ne détaillerons, dans cette partie, que la structure interne d'un robot.

\section{Architecture globale}

Chaque robot repose sur une architecture distribuée, dynamique et modulable composée de briques intelligentes (figure 2.) dialoguant sur le bus CAN. Elles viennent s'insérer dans un rack fournissant les alimentations et la connexion du bus CAN. Cette association de briques 
logicielles et matérielles autonomes (systèmes embarqués) réalisant des fonctions spécifiques autorise une mise en œuvre rapide et efficace pour des applications et cibles variées.



Figure 2. Architecture de PRoMoCo

\section{La brique Communication HF.}

Cette brique est indispensable pour des missions nécessitant la communication entre plusieurs robots et/ou avec un superviseur déporté. Elle peut s'appuyer sur différents protocoles (ZigBee, $\mathrm{WiFi}$, BlueTooth) et permet de se connecter à distance sur le bus CAN de chaque robot. Une brique de communication Zigbee/CAN est actuellement opérationnelle.

\section{La base de données}

Cette base est implantée sur un PC de bureau déporté qui peut assurer le rôle de superviseur de la flotte. Elle gère des objets de type flottes, robots, briques et fonctionnalités. Le rôle de cette base de données est de définir :

$>$ Le nombre de robots composant la flotte

La liste des briques utilisées par chaque robot

La liste des fonctionnalités de chaque brique

Cette application intègre évidemment des options de création, de suppression, de configuration des objets précédemment définis. La transmission des configurations aux robots se fait actuellement par une liaison de type ZigBee et/ou bus CAN. Ces informations peuvent ainsi être exploitées pour autoriser des possibilités d'échanges inter-robot, inter-briques, ainsi que des opérations de fusions de données pour la génération et la transmission d'informations de haut niveau. Ces activités sont indépendantes de la présence (ou de l'absence) d'un superviseur déporté ou non. Elles reposent sur la gestion de librairies unifiées de communications rapidement portables pour le développement de nouvelles briques. C'est aussi à ce niveau que sont définis les différents ID (identifiant CAN) des différentes fonctionnalités intégrées aux briques. On y retrouve pour chaque brique :

$>$ une liste de messages CAN pour chaque fonctionnalité

$>$ une définition de leur priorité (au sens du protocole CAN)

$>$ des options de transmission (sur alerte, périodique, à la demande ou autonome).

\section{L'IHM déportée}

Elle permet la génération et la réception de messages à destination de chaque brique à des fins de configuration, de supervision ou de diagnostic. Deux possibilités de connexion sont disponibles : liaison câblée sur le bus CAN des robots ou sans fils via le module «Communication HF ». Cette seconde option autorisera l'encapsulation de trames CAN dans des réseaux WiFi ou BlueTooth, autorisant l'usage d'applicatifs sur PC, sur PDA ou sur téléphone portable. 


\section{La mise en œuvre de PRoMoCo}

Afin de répondre aux différentes applications et validations de solutions industrielles et de recherche, il est indispensable que les procédures de mise en œuvre soient rapides et ergonomiques. Ces objectifs ne pouvaient être atteints que par un masquage du fonctionnement interne des briques et de leur protocole d'échanges d'informations. Pour chaque brique, nous mettons à disposition de l'utilisateur une liste de fonctions documentées en langage C. L'exécution de ces fonctions se traduit (de manière transparente pour le développeur) par l'échange de messages sur le bus CAN. Ces messages peuvent être de différentes natures :

Action de bas niveau : lecture d'une entrée de capteur, commande d'un actionneur

Action de haut niveau : lecture du cap fourni par la brique « boussole 3D »

Attente d'un événement : détection d'obstacles

Dans le dernier cas, le message CAN transmis est un message de configuration d'une brique pour un mode de transmission sur alerte ou sur seuil (voir partie II.3).

\subsection{Les briques}

\section{Structure électronique}

Le rôle principal de ces briques est de permettre un traitement d'informatique optimisée (gestion intelligente) des capteurs et actionneurs afin de réduire la charge du bus CAN : Fusion de données et transmission d'informations de haut niveau si, et seulement si, c'est nécessaire !!! .

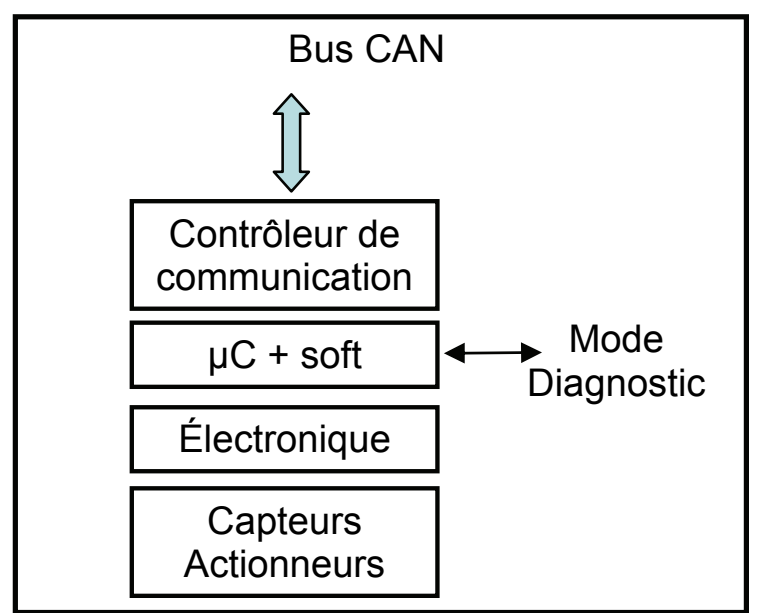

Figure 3. Architecture des briques de PRoMoCo

La figure $\mathrm{n}^{\circ} 3$ décrit la structure globale d'une brique. On y retrouve le contrôleur de communication avec le bus CAN, la partie traitement embarqué qui repose généralement sur un microcontrôleur mais ce dernier peut être remplacé par un DSP (pour la brique asservissement de trajectoire) ou par un FPGA (pour du traitement vidéo) selon les contraintes des fonctionnalités à implanter.

\section{Mode diagnostic}

Le mode diagnostic est intégré sur chaque brique pour autoriser un accès direct aux fonctionnalités de haut et de bas niveau. Afin d'être compatible avec n'importe quel type d'interfaces sur port série, la gestion et l'affichage des menus utilisateurs et des options associées sont implantés au sein même de la brique. Cette approche autorise, de ce fait, un dialogue avec chaque brique indépendamment de l'état du bus CAN et des activités des autres briques. 


\section{Options de transmission}

Pour décrire les différentes modes de transmissions disponibles sur les briques, nous nous appuierons sur la mise en œuvre de la brique « tourelle ultra sonore ». Cette tourelle a été conçue à partir d'un module télémètre à ultrasons et d'une motorisation sur $360^{\circ}$ dans le plan horizontal.

Mode autonome

Il s'agit d'un mode particulier où l'on sollicite une brique afin d'exécuter un travail plus conséquent. La tourelle accepte ce type d'ordre dans le cas d'une demande de reconstitution de l'environnement ultrasonore sur $360^{\circ}$. Les données issues de cette modélisation sont évidemment stockées en interne dans la brique et consultables par d'autres briques ou par le superviseur.

Mode transmission à la demande

Il s'agit du mode élémentaire où la brique renvoie l'information demandée une fois que son traitement est terminé : exemple mesure de distance pour un cap donné.

Mode alerte/Seuil

La tourelle peut être configurée pour balayer en continu un secteur d'angle défini avec une distance d'obstacles minimale à respecter. En cas de détection d'obstacles à une distance inférieure, un message d'alerte est transmis par la brique sur le bus CAN en précisant la direction et la distance de l'obstacle.

Mode périodique

Il consiste à configurer une brique pour fournir de façon périodique des informations sur le bus CAN : l'utilisateur demande de transmettre, par exemple, une information de mesure de distance toutes les secondes pour des directions particulières.

\subsection{Dialogue inter brique et fusion de données}

L'architecture développée pour PRoMoCo donne la possibilité à chaque brique d'identifier et de connaître les fonctionnalités des autres briques. Cette configuration autorise le développement d'algorithmes de fusion de données qui peuvent être de 3 types :

$>$ Fusion au sein d'un même robot.

$>$ Fusion entre briques de robots différents.

$>$ Fusion entre le superviseur déporté et les robots

\subsection{Historique des briques développées dans le cadre de la formation et de contrats de recherche.}

Le tableau N 1 détaille la liste des briques actuellement opérationnelles.

Carte de gestion de moteurs.

Elle repose sur l'utilisation d'un DSP Texas exploitant de manière optimale les données issues des codeurs optiques (entrée QEP). Deux boucles de régulation (vitesse et position) sont implantées dans cette carte qui peut aussi assurer la fonction de superviseur pour des missions simples.

Télécommande sécurité 
L'intégration d'un module de communication ZigBee au sein d'une manette de console de jeux permet de générer des trames CAN de déplacement sur un robot afin de reprendre la main en cas de l'exécution d'une mission défaillante mais aussi pour faciliter le déplacement des robots.

Module de communication

Ce module assure la liaison entre une brique déportée (télécommande, supervision, sécurité, robot, environnement) et les réseaux CAN des différents robots. A ce jour, les passerelles ZigBee/CAN et $\mathrm{WiFi} / \mathrm{CAN}$ ont été développées.

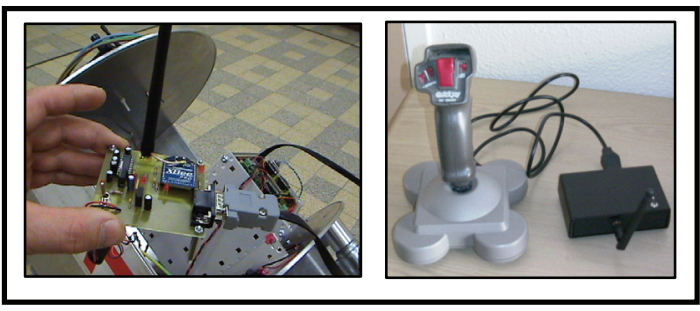

Figure 5. Passerelle CAN/ZigBee et télécommande

Conception et réalisation des bâtis mécaniques (projet étudiants IUT Génie

Mécanique de Saint-Quentin)

Carte gestion des moteurs et odométrie (asservissement vitesse et position DSP

Texas Instruments)

Librairies unifiées du Bus CAN pour famille Microchip et Texas Instruments

Opto-guidage par suivie de lignes colorées au sol.

Localisation par suivie vidéo de balises infrarouges

Positionnement par puces RFID,

Gestion de la configuration des robots (application base de données)

Gestion et recharge des batteries sans contact

Passerelle WIFI /CAN : échange entre robots et superviseur

Passerelle ZigBee/CAN : échange entre robots et superviseur

Joystick ZigBee : module commande à distance prioritaire

Tableau N 1. : Briques opérationnelles

\section{PRoMoCo : plateforme industrielle}

La seconde vocation de la plateforme est de répondre à des besoins industriels ou d'innovation technologique. Deux sociétés, spécialisées dans le développement de systèmes mobiles sans conducteur $(\mathrm{AGV})$ ont bénéficié de la plateforme.

\subsection{Guidage en milieu extérieur d'un Chariot VAO}

En 2007 nous avons validé une solution de localisation en milieu extérieur basée sur la fusion de données entre des données GPS filtrées et d'odométrie pour un chariot VAO. VAO est né d'un partenariat industriel entre BA SYSTEMES et MANITOU pour le développement et la commercialisation de véhicules «bi-modes » (manuel/automatique). Ce projet s'appuyait sur un stage étudiant, bénéficiant d'un financement de type Innova (Région Picardie). Il a obtenu le second prix SEE Nord. 


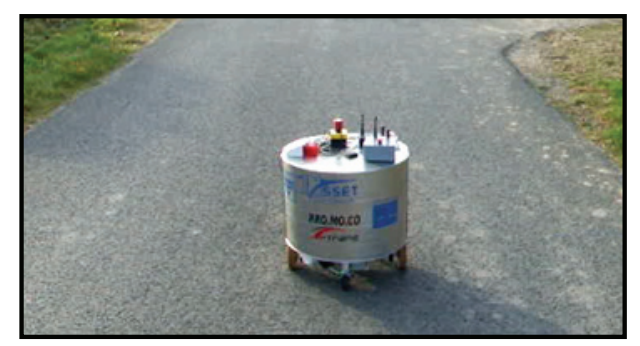

Figure 5. Essai en extérieur de localisation GPS/odométrie

\subsection{Solution de guidage optique par technologie RFID}

En 2008 nous avons développé une brique de guidage basée sur la détection et l'identification de tag RFID insérés dans le sol, associée à un dispositif existant de suivi de ligne. Cette brique autorise des passages robustes entre le mode de conduite manuel et le mode de conduite automatique. Ce projet concernait la phase d'industrialisation du chariot C30-VAO de la société Manitou. Il a été par la suite présenté au salon mondial de la logistique à Hanovre (Stage M1 SET, financement INNOVA Région Picardie).

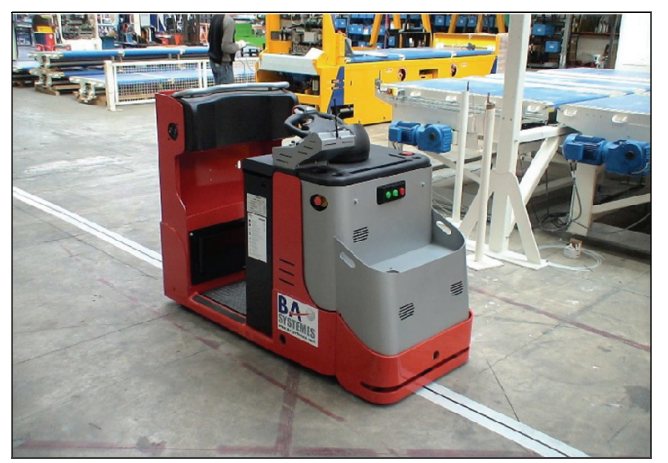

Figure 6. Phase de validation dans les locaux de la société BA Systèmes (Rennes)

\subsection{Etude de faisabilité et développement d'une brique de localisation basée sur un télémètre magnétique pour le guidage d'un AGV}

La problématique traitée concernait le développement d'un système de localisation absolue d'un robot mobile à partir de la détection d'aimants insérés dans la zone d'évolution. Elle s'appuie sur l'utilisation d'un ensemble de boussoles 3D pour la mesure précise du champ magnétique des aimants et du champ magnétique terrestre. Afin de tenir compte des contraintes d'intégrations variées selon les mobiles et de conserver les avantages de l'architecture modulaire de PRoMoCo, cette brique de localisation est composée de "sous briques » dialoguant sur un bus CAN spécifique. 


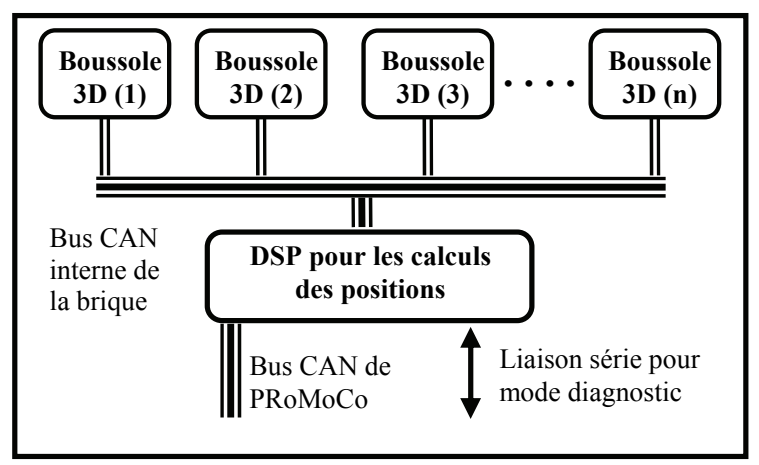

Figure 7. Brique de localisation composée d'autre briques

Cette brique de guidage procure de nombreux avantages car elle est peu coûteuse, robuste, autorise des transitions intérieur/extérieur et tient compte des contraintes de mise en place aisée et discrète lors de l'installation des balises pour certains environnements (hôpitaux, cave à vins) car les aimants sont enterrés dans le sol.

\section{Conclusion}

\subsection{Le bilan}

La plateforme PRoMoCo répond tout à fait aux objectifs que nous avions définis. Elle autorise une mutualisation des investissements humains (étudiants, équipe enseignante, chercheurs) et financiers (formation SET, contrats industriels et projets européens INTERREG). Elle constitue un élément essentiel de mise en application des disciplines de notre formation systèmes embarqués (plus de $80 \%$ des projets proposés aux étudiants concernent le développement de «briques » pour PRoMoCo). Elle est très rapidement opérationnelle pour des démonstrations d'intégration de nouvelles technologies ou de validation de prototypes industriels. C'est aussi une vitrine de promotion de nos activités de recherche que nous présentons régulièrement lors de salons, colloques des laboratoires, ou de manifestations scientifiques (Printemps de l'Industrie, Salon Européen INNOVACT).

\subsection{Perspectives}

Afin d'ouvrir la plateforme aux laboratoires et aux entreprises géographiquement distants, un serveur Web (passerelle Internet Bus Can) sera mis en place pour autoriser une gestion complète et sécurisée via Internet. Les librairies de gestion du bus CAN seront réécrites pour l'intégration du nouveau protocole de communication automobile Flexray.

Cette plateforme va aussi s'enrichir d'un nouveau robot mobile (en collaboration avec l'IUT de mécanique) qui sera équipé de 4 roues motrices indépendantes et d'une motorisation de type brushless. Nous traiterons aussi des problématiques de l'optimisation de la recharge des batteries au moyen d'énergie solaire en coopération avec d'autres laboratoires de l'Université. PRoMoCo sera tout également la plateforme d'expérimentation des solutions qui seront développées dans le cadre d'un projet de recherche déposé dans le pôle de compétitivité ITRANS [6] sur la problématique du chargement automatique de containers. 
[1] www.insset.u-picardie.fr

[2] http://mis.u-picardie.fr/

[3] Capitaine T., Venet T ., Senlis J., "Use of DSP technology for improving our Orbital System" IEEE ISIE 2004 International Symposium on Industrial Electronics in Ajaccio, France 4th to 7th May, 2004.

[4] P. Carton, T. Capitaine, V. Bourny, J. Senlis: European Project Interreg III "Réseau Ressources Formations Industries (2RFI)", IEEE ICELIE 2006, International Conference on ELearning in Industrial Electronics, December 18-20, 2006 -Hammamet, Tunisie

[5] Capitaine T., V.Bourny Senlis J., Hamzaoui Projet européen Interreg III "Réseau Ressources Formations Industrie (2RFI)" - Génération de trajectoire d'un robot mobile, CETSIS 2005, Nancy [6] http://www.i-trans.org/

\section{LES AUTEURS}

Valéry Bourny est Maître de conférences à l'Université de Picardie Jules Verne (UPJV) en Picardie, en poste à 1 'INstitut Supérieur des Sciences Et des Techniques (INSSET) de SaintQuentin. Il est responsable pédagogique du niveau M1 de la formation SET et membre du laboratoire MIS (Modélisation, Information et Systèmes) dans l'équipe PR (Perception en Robotique).

Thierry Capitaine est Maître de conférences à l'INSSET/UPJV. Il est à l'origine de la création et le responsable du Master Systèmes Embarqués dans les Transports (SET). Il est membre du laboratoire MIS dans l'équipe PR (Perception en Robotique).

Jacky Senlis est professeur agrégé en physique appliquée en poste à l'Insset /UPJV. Il est responsable pédagogique du niveau M2 de la formation SET.

Mohamed Hamzaoui est Maître de conférences à l'INSSET/UPJV. Il est responsable pédagogique du niveau L3 de la formation SET.

Aurélien Lorthois est assistant ingénieur à l'INSSET/UPJV.

Ludovic Duval est assistant technicien à l'INSSET/UPJV. 